\title{
Atypical Presentation of Corneoscleral Granuloma in a Pug and its Surgical Management
}

\author{
Sarbani Hazra ${ }^{1}$, Aditya Konar ${ }^{2}$ and Subramanian Krishna Kumar ${ }^{3}$ \\ ${ }^{1}$ Dept of Veterinary Surgery \& Radiology, West Bengal University of Animal \& Fishery Sciences, Kolkata \\ ${ }^{2}$ CSIR-IICB, Jadavpur, Kolkata \\ ${ }^{3}$ Sankara Nethralaya, Institute for Research in Vision and Ophthalmology, Chennai, India
}

Received: 13 April, 2017; Accepted: 23 Aug, 2017; Published: 29 Aug, 2017

*Corresponding author: Sarbani Hazra, Dept of Veterinary Surgery \& Radiology, West Bengal University of Animal \& Fishery Sciences, Kolkata, India. E mail: shazrakon@yahoo.co.in

\begin{abstract}
A 2-year-old intact male pug was presented to the clinics at West Bengal University of Animal \& Fishery Sciences with a complaint of rapidly growing growth on the corneoscleral junction of the ocular surface. A routine ophthalmic examination was performed. Decision to perform a surgical resection of the growth was taken. The growth was removed under general anaesthesia, and the excised mass was subjected to histopathological examination. Postoperative management was done with antibiotics, systemic as well as topical drops, anti-inflammatory and cyclosporine drops. Histopathological examination identified the mass as an inflammatory granuloma. The wound healed by one week but a scar was evident on the corneal surface. Peripheral location did not affect the visual axis. Clinical presentation of such a rapid growing, aggressive ocular surface granuloma has not been reported earlier in a pug.
\end{abstract}

Keywords: Nodular granuloma; Pug; Histopathology

\section{Introduction}

Canine Ocular Nodular Episcleritis (CONE) refers to a condition presented as a fleshy elevated lesion occurring generally on the limbus or sclera, though other sites of an occurrence like third eyelid, eyelids and even retro bulbar space have also been reported. It is usually unilateral in presentation and affects middle- aged, both male and female dogs.

There is yet no defined literature on its etiopathogenesis [1,2]. Therefore more case reports of clinical and histopathological presentation are required to add to existing literature to arrive at the identification of possible causes of its pathogenesis.

Although the nodular granulomatous form of episclerokeratitis is commonly reported in collie, We hereby present a case report of an atypical corneoscleral granuloma in a two year old male pug $[2,3]$. The unusual presentation of this case was the young age of occurrence and the aggressive nature of the growth.

\section{Materials \& Method}

A 2-year-old intact male pug was presented with an aggressive growth on the corneoscleral junction (Figure 1A). Detailed ophthalmic examination i.e., slit lamp bio microscopy, schirmer tear test, fluorescein dye test, direct and indirect ophthalmoscopy was performed in both eyes to rule out other abnormalities if any. A decision for surgical removal was planned, and preoperative blood examination was advocated. The patient was premedicated with atropine sulphate @.02mg/ kg and xylazine $\mathrm{HCl} 1 \mathrm{mg} / \mathrm{kg}$; Anesthesia was induced with a combination of ketamine HCL @5 mg/ kg and diazepam@0.2mg/kg. Surgery was performed under an operating microscope. After aseptic preparation of surgical site with drape and painting with povidone iodine, an eyelid speculum was placed to retract the eyelids. The growth was held with a conjunctival forceps, the base was broad and encroached the limbus and invaginated into peripheral corneal surface up to about $2 \mathrm{~mm}$. A no 11 scalpel blade was used to incise the growth from the base carefully by not penetrating deep into cornea (Figure 1B). There was moderate bleeding; the tissue felt hard and brittle. The excised tissue was preserved in $10 \%$ formaldehyde for histopathological investigation. Local hemostasis was achieved by swabs soaked in 1:10000 adrenalin. The patient was prescribed a systemic antibiotic (cefotaxime) for five days post surgery and anti-inflammatory for three days (meloxicam). Topical drops of anti-inflammatory, antibiotic and immunosupressent flurbiprofen, moxifloxacin and cyclosporine respectively were prescribed for two weeks post operatively. The patient was advised the use of an E-collar for up to two weeks following surgery.

\section{Results}

The blood reports indicated that the patient was physiologically stable. The affected eye had mild scarring and melanosis on the cornea, anterior to the growth. There was no other abnormality, tear formation and corneal integrity, anterior chamber and fundus were normal. The contra lateral unaffected eye was normal.

The patient had a smooth recovery from anaesthesia, and there was no intraoperative complication. One week after surgery the surgical bed was slightly edematous, two weeks following surgery the surgical bed had completely healed with a scar tissue evident on the affected site (Figure 1E). A follow-up period of 4 months showed no recurrence of the growth.

\section{Histopathology}

The sections of the tissue stained with H\&E showed normal appearance of epidermis while the dermis showed dense nodular 


\section{Management}

granulomatous inflammation. The presence of histiocytes, occasional multinucleated giant cells and dense collections of lymphocytes and plasma cells were evident. Scattered tiny blood vessels and numerous spindle-shaped cells mixed with histiocytes, lymphocytes and plasma cells were identified. Also, the tissue was negative for fungus. (Figure1C and 1D)

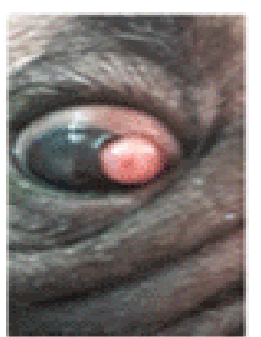

A

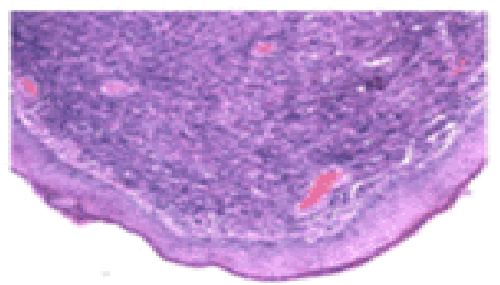

$\mathrm{C}$

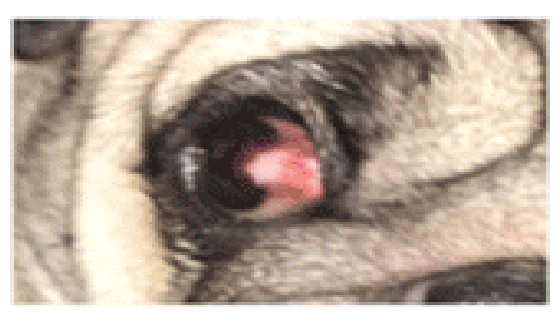

E

Figure 1:

A.Shows the clinical presentation of a two year old pug with rapidly growing nodular granuloma on the corneoscleral junction.

B.Intra-operative image of wound bed following excision of the nodular growth. C. The sections of the tissue stained with H\&E shows normal appearance of epidermis while the dermis showed dense nodular granulomatous inflammation. D.The sections of the tissue stained with H\&E shows, presence of histiocytes, occasional multinucleated giant cells and dense collections of lymphocytes and plasma cells are evident. Scattered tiny blood vessels and numerous spindle-shaped cells mixed with histiocytes, lymphocytes and plasma cells are identified.

E. Post operative recovery shows complete healing of the wound with scar evident on the cornea.

\section{Discussion}

Canine ocular nodular episcleritis is an infrequent presentation. The etiopathogenesis of the condition is not clearly defined. Their clinical presentation is also varied; it is either diffuse or nodular in nature. Some breeds show the predilection for diffuse episcleritis i.e., cocker spaniel and golden retriever, while the nodular presentation is common with the Collie [3]. Middle aged dogs of either sex are most commonly affected. In the present case report, we present the incidence of nodular granuloma in a young male pug, both the age and breed, have not been reported as a common occurrence in the previous studies. Consistent with the previous incidence of $90 \%$ episcleral granuloma occurring unilateral, in this case too, the lesion was unilateral, although bilateral occurrence due to systemic infection has been reported [4]. The aggressive fleshy nodular growth in the limbus can be described as episclerokeratitis, and the histopathological findings corroborate with previous reports [3]. There was no evidence of fungal or bacterial infection; it was therefore assumed to be of autoimmune origin, which is a major predisposing factor for this condition [5]. Although a conservative treatment with immunosuppressive is a routine approach, in this case, surgery was planned owing to the rapid nature of the growth.

The surgery was successfully performed, and immunosuppressive drug cyclosporine was prescribed in addition to other mainstay therapeutics to control recurrence, a follow-up period of up to four months showed no recurrence, scarring on the cornea was observed as a sequel. Previous study with such an atypical nodular episclerokeratitis failed to respond to systemic and topical immunosuppressive therapy and eventually lead to loss of vision and enucleation [6,7]. As case reports on nodular episclerokeratitis in dogs is scarce. This case report puts forth surgical management of nodular granuloma in a pug and its histopathological findings.

\section{Acknowledgement}

We acknowledge the facilities and support received from WBUAFS for conducting the study.

\section{References}

1. Deykin AR, Guandalini A, Ratto A. A retrospective histopathologic study of primary episcleral and scleral inflammatory disease in dogs. Vet Ophthalmol. $1997 ; 7(4): 245-248$

2. Dungan SJ, Ketrig KL, Severin GA, Render JA. Variant nodular granulomatous episclerokeratitis in four dogs. J Am Hosp Ass. 1993;29:403-409.

3. Paulson ME, Lavach JD, Snyder SP, Severin GA, Eichenbaum JD.Nodular granulomatous episclerokeratitis in dogs: 19 cases. J Am AnimHosp Assoc. 1987;190(12):1581-1587

4. Fischer CA. A clinicopathologic classification of episcleritis and scleritis in the dog. ProcAm Coll Vet Ophthalmol. 1982;13:1

5. Willis M, Martin CL, Stiles J. Infectious diseases as a possible cause of scleritis and episcleritis. Transactions of the annual scientific programs of the American VeterinaryCollege of Ophthalmologists. 1996;27:45

6. Ketring, KL, Glaze, MB. Nodular episcleritis Atlas of breed-related canine ocular. Disorders. SBN : 1884254446 Record Number : 19982220275 Publisher : Veterinary Learning Systems, 425 Phillips Boulevard.Location of publication : Trenton, NJ 08618Country of publication : USA. 1998;140

7. Kim TH, Jeong MB, Park S, Seo KM. A typical nodular granulomatous episclerokeratitis in a dog. Journal of Veterinary Clinics. 2010;27(1):102-106. Legends 